\title{
The Effectiveness of Minor Procedure as the Management of Onychocryptosis in Community Podiatry Setting: A Retrospective Study
}

Shu Xin Teh ( $\nabla$ shuxin-96@hotmail.com )

University of Huddersfield https://orcid.org/0000-0001-7634-4246

\section{Andrew Bridgen}

University of Huddersfield

Shek Hong Ip

Frimley Park Hospital NHS Trust: Frimley Health NHS Foundation Trust

\section{Research}

Keywords: Management of onychocryptosis, Nail surgery with phenolisation, Recurrence rate following nail surgery, Postoperative infection rate

Posted Date: December 28th, 2021

DOI: https://doi.org/10.21203/rs.3.rs-1159053/v1

License: (a) (1) This work is licensed under a Creative Commons Attribution 4.0 International License. Read Full License 


\section{Abstract}

Background: Onychocryptosis is a common and debilitating condition that often require surgical management. Despite this minor procedure being the most common and effective method in managing this condition, as well as being one of the essential services provided by Health Care Professional Council (HCPC) registered podiatrists, there is limited evidence around the effectiveness of this procedure in the community setting. The aim of this study was to evaluate the effectiveness of a non-invasive minor procedure for the management of onychocryptosis.

Methods: A retrospective study was undertaken within the community podiatry department. All patients who underwent minor procedure for the management of onychocryptosis between June 2018 and December 2019 were included in this study. The data were collected from the electronic patient records using a comprehensive data collection tool. Recurrence rate and infection rate were calculated to determine the effectiveness of the minor procedure.

Results: 354 minor procedures were performed on 272 patients presenting with onychocryptosis. The recurrence rate of nail regrowth following intervention was $5.6 \%(\mathrm{~N}=20)$. Patients $<40$ years old were more likely to experience a recurrence of nail regrowth following an intervention $(\mathrm{N}=16)$. The postoperative infection rate was $8.8 \%(\mathrm{~N}=31)$. Patients who had a partial nail avulsion procedure were more likely to experience postoperative infection compared to total nail avulsion.

Conclusions: Although the management of onychocryptosis with this minor procedure is effective within the community podiatry department, incorporation of evaluation of patient satisfaction following this procedure is suggested within the recommendations. The limitations of this study have been highlighted and a mixed method research project is recommended to explore this area of specialty to improve patient outcome.

\section{Background}

Onychocryptosis, known colloquially as ingrown toenail, is a common condition in adults and children, affecting $2.5-5 \%$ of the general population (1). It is reported to be accountable for $21.7 \%$ of the conditions that were treated by podiatric surgeons in a retrospective cohort study in Australia (2). The prevalence of this condition is higher in males and among adolescents (3). This could be attributed to the increased chances of sustaining minor trauma to the feet as a result of a greater physical activity level (4). This condition often causes pain, psychosociological distress and affects activities of daily living. There is subsequently an increased risk of falls especially in the elderly (5). The development of onychocryptosis is reported to be multifactorial, including poor nail cutting technique, nail morphology, hyperhidrosis, poor footwear, structural foot deformities, systemic diseases, trauma and obesity $(3,6)$. Furthermore, it has also been reported that the prevalence of onychocryptosis is higher in diabetic patients, with the frequency of unilateral onychocryptosis, especially in the lateral margin, being more common as a result of increased pressure during the gait cycle (1). They also highlighted that patients with endocrine disease 
with concomitant cardiovascular disease portrayed a higher risk at developing onychocryptosis than those without cardiovascular disease, which can be explained by both micro and macrovascular complications along with endocrine disorders potentially affecting the nutritional supply of the nail unit.

There are a number of different methods have been described for the treatment of onychocryptosis, ranging from simple conservative approaches to surgical procedures (7). A systematic review and metaanalysis concluded both nonsurgical and surgical interventions yielded high levels of patient satisfaction. However, these findings may not be universal as there is a lack of an accepted standardised method for reporting patient outcomes and satisfaction (8). Conservative approaches include silver nitrate cauterization of the granulation tissue, gutter treatment and orthonyxia (7). These treatments are deemed to require a high level of patient concordance and time. Surgical management is often indicated if there is presence of recurrent infection or when conservative measures have been exhausted (9). A wide range of incisional and non-incisional surgical methods are available for treatment of onychocryptosis; however, there is no evidence that any single technique is the procedure of choice in all cases (7). Matricectomies are frequently performed in both surgical methods to achieve a high success rate (7). This can be performed either surgically or using chemical agents, such as trichloroacetic acid, sodium hydroxide or phenol with the advantage of faster healing time, which is desirable especially in at-risk group patients $(10,11)$. Phenol is an effective protein denaturant, whereby the property of cauterisation works by producing a coagulation necrosis in the matrix and the surrounding soft tissue (12). Phenol usage has been shown to increase the effectiveness of surgical intervention in preventing recurrence of the symptomatic nail (7). Although phenol is known to be contraindicated for both clinicians and patients who are pregnant as there is a risk of local and systemic toxicity, phenol remains the premier agent for chemical matricectomy due to it having the most research and thus subsequent knowledge base (10). Furthermore, it has strong antiseptic properties and acts as a postoperative analgesic secondary to the demyelination of the terminal nerve endings (13).

Nail avulsion with phenol matricectomy is a common procedure as it is a highly effective treatment with low recurrence rates (14). Recurrence is defined as a clinical reappearance following the procedure within the minimum 6-months follow-up period (7). This is an important indicator as to the success of the procedure in providing permanent resolution of onychocryptosis (15). However, there is no consensus on the correct duration of phenol application, which varies between 30 seconds to 4 -minutes (16). A prospective randomized trial of 110 patients comparing the efficacy and safety profile of different durations of $88 \%$ phenol application suggested that there is no significant difference between 1-minute, 2-minutes or 3-minutes phenol application with respect to recurrence rate, with all the recurrences observed within 6 months $(p=0.092)(17)$. This finding is contradictory to an observational study of 422 patients which reported that a total of 4-minutes of continuous phenol application appears to be appropriate to achieve optimum outcomes (recurrence rate of 1.1\%) (16). As a result of the number of conflicting studies, there is no definitive duration of phenol application that has been agreed upon as being strictly superior to another. 
A recent systematic review highlighted that the rate of postoperative surgical site infections following foot and ankle surgery ranges between $0-9.4 \%$ and that the use of antibiotic prophylaxis decreases the infection rate (18). The risk factors for postoperative infection in surgical management for onychocryptosis includes age, systemic diseases and surgery settings (2). Despite the evidence supporting phenol to be used for this procedure, there are conflicting opinions as to whether phenol may increase the risk of subsequent infection $(6,19)$. Several studies have reported that partial nail avulsion with phenolisation increases the risk of postoperative infection, citing associated tissue damage and increased postoperative bleeding compared to other cauterization agents $(19,20)$. Conversely, there are also studies that find phenolisation does not increase the risk of postoperative infection $(13,14)$. The contradicting results could be explained by the different methodologies used in these studies, including varying concentrations of phenol used, different duration of phenol application, postoperative dressing regime and heterogeneity in surgical methods. Therefore, despite the fact that phenol usage is essential in reducing rates of recurrence, there remains no consensus with regards to the risks of potential concomitant complications, which in themselves are not negligible.

\section{Methods}

The present study aims to identify the effectiveness of this minor procedure within the community podiatry department. This study was carried out retrospectively within a community podiatry department setting using direct observation of patient records from EMIS (a validated, centrally hosted clinical computer system employed at the practice).

\section{Recruitment of participants}

Convenience sampling is used to select patients for this study. Ethical approval from the University School Ethics Panel and permission was given by the Trust for the use of the retrospective anonymised data.

\section{Inclusion/Exclusion Criteria}

Inclusion Criteria

- Surgical indication as a treatment for onychocryptosis.

- Patients who underwent minor procedure between 1st of June 2018 - 31st of December 2019.

\section{Exclusion Criteria}

- Revision procedure that involves removing a portion of nail that is a regrowth following previous unsuccessful surgery that resulting in persistent symptoms.

- Treatment indication for other nail pathologies; for example, onychomycotic nails, traumatic nail injuries, onychauxic nails. 
- Procedures with elective decision to opt out phenol used as this may skewed the results due to the high recurrence rates without phenolisation has been discussed in the literature (7).

\section{Data Collection}

A comprehensive data collection tool is essential to capture the required information to answer the research question yet must also be precise enough to avoid generating extensive amount of irrelevant data. The design of the data collection tool, using Microsoft Excel Spreadsheet was undertaken with the support from an analyst from the local Trust. This support from the analyst ensured that the collection tool correlated to the aims and objections of the study, to limit subjectivity and to reduce observer bias, enhancing the validity and reliability of the results (21). The list of patients who attended for the minor procedure between $01 / 06 / 2018$ to $31 / 12 / 2019$ was generated automatically using the auto report function in the patient record system. Demographic details, such as gender, and age are also included from the auto report. The demographic details are then transferred to the data collection tool (Appendix 1). The growth of an asymptomatic nail spicule is considered as postoperative sequalae, which in this study, will not be categorised as recurrence (22). Postoperative infection was identified by the postoperative follow-up documentations; if there were any signs of infection present or antibiotics was clinically indicated. Once the collection tool was established, data was extrapolated from patient healthcare records.

\section{Data Analysis}

Descriptive statistics will be used to outline the characteristics of the study group. Measures of central tendency (mean, mode, median) will be used to describe the data for the results obtained.

\section{Results}

A total of 395 minor surgeries were performed between 01/06/2018 to 31/12/2019; of which 354 procedures treated on 272 patients were included in this study following the inclusion and exclusion criteria (Figure 1).

\subsection{Demographics of participants}

The baseline characteristics of the study population are shown in Table 1 and Figure 2. 
Table 1

Characteristics of included procedures

\begin{tabular}{|c|c|c|c|}
\hline Characteristics & \multicolumn{3}{|c|}{ All subjects $(\mathrm{N}=354)$} \\
\hline \multicolumn{4}{|l|}{ Gender, n (\%) } \\
\hline Male & $201(56.8 \%)$ & & \\
\hline Female & $153(43.2 \%)$ & & \\
\hline Age, n (\%) & & Male, n (\%) & Female, n (\%) \\
\hline $0-18$ & $85(24.0 \%)$ & $61(17.2 \%)$ & $24(6.8 \%)$ \\
\hline $19-40$ & $94(26.6 \%)$ & $54(15.3 \%)$ & $40(11.3 \%)$ \\
\hline $41-64$ & $89(25.1 \%)$ & $34(9.6 \%)$ & $55(15.5 \%)$ \\
\hline$>65$ & $86(24.3 \%)$ & $52(14.7 \%)$ & $34(9.6 \%)$ \\
\hline \multicolumn{4}{|c|}{ Concomitant diseases, n (\%) } \\
\hline Yes & $107(30.2 \%)$ & $65(18.4 \%)$ & $42(11.9 \%)$ \\
\hline No & $247(69.8 \%)$ & $136(38.4 \%)$ & $111(31.4 \%)$ \\
\hline \multicolumn{4}{|c|}{ Social history, n (\%) } \\
\hline None reported & $329(93 \%)$ & $190(53.7 \%)$ & $139(39.3 \%)$ \\
\hline Smoking & $25(7 \%)$ & $11(3.1 \%)$ & $14(3.9 \%)$ \\
\hline
\end{tabular}

\section{Procedures performed}

Among these 354 procedures, 195 were carried out as a single treatment; 72 participants had 2 procedures performed within 1 appointment session contributing to $40.7 \%$ of the procedures $(N=144) ; 5$ participants had 3 procedures performed within 1 appointment session, contributing to $4.2 \%$ of the procedures in this study $(\mathrm{N}=15)$.

Total nail avulsion was performed in $15.3 \%$ of procedures $(\mathrm{N}=54)$; whereas partial nail avulsion was performed in $84.7 \%$ of procedures $(\mathrm{N}=300)$. Interestingly, for unilateral partial nail avulsion, there is a higher incidence of the procedures performed on fibula aspect only compared to the tibial aspect (Figure 3). All the procedures had a duration of $2 \times 1$ minute liquid phenol $89 \% 0.15-0.20 \mathrm{ml}$.

\section{Recurrence of nail regrowth}

Among the 354 procedures performed, recurrence of nail regrowth was recorded in $5.6 \%$ of the sample $(\mathrm{N}=20)$. Of all the procedures that represented with recurrence $(\mathrm{N}=20), 10 \%$ were total nail avulsions $(\mathrm{N}=2)$ and $90 \%$ were partial nail avulsions $(\mathrm{N}=18) .14$ procedures with recurrence were performed as a single procedure in one appointment; 6 procedures were performed as a multiple procedure in one appointment. 
The range of months taken to observe recurrence of nail spike was documented between 1-22 months postoperatively (Figure 4). The mode of nail recurrence observed is 3 , which happened in the $2 \mathrm{nd}$ and 12th months postoperatively. The median time taken for recurrence of nail regrowth is 8.5 months postoperatively. All the cases with recurrence $(\mathrm{N}=20)$ decided to have a revision procedure to remove the offending portion.

In the subgroup which presented with recurrence, the mode age group is between 19-40 years old ( $N=9)$ (Figure 5). There is a higher incidence of females $(\mathrm{N}=13)$ compared to males $(\mathrm{N}=7)$ for having a recurrence following the procedure. $90 \%$ of the patients $(\mathrm{N}=18)$ with a recurrence were in general good health without any prescribed long-term medication. $10 \%$ of the patients $(\mathrm{N}=2)$ had medical conditions, which were endocrine disorders and cardiovascular disease.

\section{Postoperative infection}

Among 354 procedures performed, postoperative infection was documented in $8.8 \%$ of patient's records $(\mathrm{N}=31)$ following intervention. All these participants received antibiotics following clinical suspicion of postoperative infection. Of 31 procedures with postoperative infection, $19.4 \%$ of the procedures $(\mathrm{N}=6)$ were a total nail avulsion; $80.6 \%$ of the procedures $(\mathrm{N}=25)$ were a partial nail avulsion procedure. 21 procedures identified as having postoperative infection were performed as a single procedure in one appointment. The time taken to observe an infection postoperatively ranged between 2 to 6 weeks.

In the subgroup with identified postoperative infection ( $N=31)$, the mode was in the 41-64 age group $(\mathrm{N}=16)$ (Figure 6).

Among all procedures that presented with postoperative infection, $48.4 \%$ of participants $(N=15)$ were in general good health without regular medications prescribed. $51.6 \%$ of the procedures $(N=16)$ had relevant medical conditions that potentially contributed to postoperative infection (Figure 7). Of all the procedures that presented with postoperative infection, only one patient reported having a history of smoking; other patients reported no social factors that could potentially influence the rate of healing, for instance: alcohol abuse, illicit drug-use. Interestingly, of all procedures with postoperative infection $(\mathrm{N}=31)$ and recurrence $(\mathrm{N}=20)$, only 4 procedures with postoperative infection presented with a recurrence as well.

\section{Discussion}

The results of this study demonstrate the relative effectiveness of a minor procedure within the community podiatry setting over a period of 18 -months. The incidence of onychocryptosis was found to be higher in males than females before the age of 40 years. This finding is similar to an epidemiology study which reported that onychocryptosis was observed to be more prevalent in men under 30 (23). This may be explained by another study which found that the incidence of onychocryptosis is higher in young males who are physically active (4). Furthermore, the nail growth is greater in males than females until the age of 70 and the situation reverses after this point which may account for the findings $(24,25)$. $15.8 \%$ of the procedures included in this study involved a patient deemed to have an endocrine and 
metabolic disorder, which correlates with a previous study which reported that the prevalence of onychocryptosis was found to be greater in patients with diabetes mellitus compared to a healthy population (1). Moreover, the frequency of unilateral onychocryptosis, (fibular>tibial) was congruent with previous research which highlighted similar findings (1).

A Cochrane review concluded that the addition of phenol increases the effectiveness of this minor surgical intervention by preventing recurrence and regrowth of the symptomatic nail (7). This is further supported by a recent systematic review comparing the efficacy of chemical matricectomy using trichloroacetic acid, phenol and sodium hydroxide which concluded that the success rate of using phenol as matrix cauterization is high, with its limitations being that it is contraindicated for patients or clinical practitioners who are pregnant (10). 10 procedures within this study were excluded due to their elective decision to not have phenol chemical matricectomy. $90 \%$ of the sample population who declined phenol application were females $(\mathrm{N}=9)$ citing personal and cosmetics reasons. These figures are lower comparing to the annual report by PASCOM-10 which found that there was a rate of $0.32 \%(N=68)$ with regards to procedures performed without phenolisation in 2018 and $0.24 \%(\mathrm{~N}=49)$ procedures in 2019 $(26,27)$. Nonetheless, it was decided in this study to exclude the procedures without phenolisation for data analysis because this may influence the results, especially in recurrence rate.

The rate of recurrence found in our study is $5.6 \%(\mathrm{~N}=20)$; as compared to the data reported by PASCOM10 , which is summarised as a $0.22 \%$ rate of recurrence $(\mathrm{N}=8)$ recorded in 2018 , and $0.26 \%$ of cases $(\mathrm{N}=11)$ in $2019(26,27)$. This discrepancy requires exploration for potential explanation. One potential factor may be due to overall number of total treatments. The rate of recurrence in the national report is based on the total number of treatments carried out annually, which was 14872 in 2018 and 14685 in 2019 across 113 centres across the country. All 20 cases of recurrence in this study decided to have a revision procedure. Asymptomatic recurrences were classified as postoperative sequalae rather than recurrence (28). With regards to comparable studies in a community setting, a published audit reported a $1 \%(\mathrm{~N}=1)$ rate of nail spike regrowth (9). This was defined as an incident which required revision surgery. However, with regards to participants involved, Krishan et al.'s study uses American Society of Anaesthesiologists (ASA) physical classification system to classify comorbidities. $10 \%$ of the participants $(\mathrm{N}=8)$ were ASA grade 2 without clear definitions of qualifying medical conditions. As a consequence, due to the small nature of their study and the lack of strong participant criteria, comparisons are made difficult, with the result that a true baseline is uncertain. Additionally, the lack of corroborating data and studies make conclusions difficult to validate for practice implications. The lower rate of recurrence in Krishnan et al.'s study may be further explained by the additional minute of phenol application. However, this explanation is contrary to the finding of a randomised trial which concluded that there was no statistically significant difference in recurrence rate between 2 minutes and 3 minutes of phenol application ( $p=0.092)(17)$. Whilst Tatlican and colleagues have established that their groups are randomised, and that there is no statistically significant difference between the disease durations among groups $(p=0.185)$ therein, the randomisation method is not specified. Moreover, although they stated there is no statistically significant difference, it is hard to ignore how there nevertheless seemed to be a correlation between disease duration and duration of phenol application. Fundamentally, 1 minute of 
phenol was applied on patients with shorter average disease duration (5.8 months), whereas 3 minutes phenol was applied on patients with an average of 7 months disease duration, which affects the validity of the construction of Tatlican et al.'s study. Furthermore, the similar issue of comparison population arises, where the participants included did not have any declared comorbidities. The implications of this practice are that the findings of this study may not be extrapolated to other populations and as a direct comparison to our study.

The duration of phenol application has been identified as one of the contributing factors to the effectiveness of the minor procedure. The latest nail surgery guidelines published by the Royal College of Podiatry in February 2019 recommended a 3x1 minute application, whereby all the procedures within our study had a 2x1 minute application (3). Although the guidelines recommended 3x1 minute phenol application, the evidence for this recommendation was questionable as the papers included did not recommend a definitive phenol application time $(7,29)$. The reliability of the evidence used in the guidelines should be questioned due to the majority of the evidence base cited being three or less in the hierarchy of evidence (29-31). The recent systematic review did not provide any recommendation on phenol application time but has made recommendation for research in this aspect based on its review on the limited evidence (7). It is worth highlighting that there is only one piece of research in this systematic review that investigated in the effectiveness of different phenol duration (17). Moreover, Tatlican et al.'s study did not include a control group and the construct validity is questionable with a possible risk of selection bias. To date, there appears to be a lack of an agreed upon standard with regards to the duration of phenol application internationally.

A histology study of 30 fresh cadavers concluded that a 4-minute application of $88 \%$ phenol solution is sufficient to achieve complete destruction of germinal nail matrix (32). Although their study found that the application time is directly proportional to the depth of burn, their study also found that the cauterization effect was not as significant when application length was over five minutes (32). This correlates with a much earlier postulation that suggested precipitated proteins prevent further phenol penetration via formation of a coagulated protein barrier (33). Transferring this finding to clinical practice, a 4-minute continuous phenol application yielded a low recurrence rate of $1.1 \%$ in an observational study of 622 patients within 1-year follow-up period (16). However, the high success rate may be explained by the variation of surgical methods used in Montesi et al.'s study where they did not eliminate the excess phenol with alcohol. This concept is supported by an experimental study that the alcohol lavage step after phenolisation is effective in diluting the residual phenol that could potentially contribute to continuous caustic effect on the germinal nail matrix (34). Another randomised, double-blinded trial of 84 patients found that a 4-minutes continuous application of phenol yielded a $100 \%$ success rate within a 4months follow-up period (13). However, the construct validity of Andre et al.'s study is contentious as the follow-up period was only 4 months, as other studies have reported that the recurrence can happen anytime between 11-17 months $(35,36)$. Furthermore, the volume of the phenol used could be another cofounding factor. It should be acknowledged that both Montesi et al.'s and Andre et al.'s studies used one phenol ampoule for 4-minutes continuous application. In our study two phenol ampoules were used, with each being applied for one minute, despite the fact that the ampoules were the same, which contains 
$0.15 \mathrm{ml}-0.20 \mathrm{ml}$ of $89 \%$ phenol $(13,16)$. As there is significant disparity among the literature, further highquality research is required in this area.

Interestingly, the majority of the cases of recurrence were in people under 40 years of age, which is similar to findings by an observational study which found that older age may be a protective factor against the recurrence of onychocryptosis when treated with phenolisation (16). A possible explanation is that nail growth decreases with age (37). Furthermore, among the procedures which presented with a recurrence, $10 \%$ of them $(\mathrm{N}=2)$ had an underlying medical condition, which were cardiovascular diseases and endocrine disorders. This finding correlates with a previous study which found that there was a statistically significant relationship between cardiovascular disease and recurrence risk $(p=0.014)(16)$. The clinical implication of this finding is that patients with cardiovascular disease may potentially require a longer duration of phenol application to achieve a better outcome. However, this should be considered in the context of the risk of postoperative infection in this group of patients with delayed healing, and in itself would benefit from further trials.

The rate of postoperative infection observed in our study was $8.8 \%(\mathrm{~N}=31)$. This figure is higher compared to a published audit completed by another community podiatry department which reported a $3 \%$ rate of surgical site infection (9). This may be explained by the significant differences in sample population between both studies. Due to small sample size, the figures may be skewed as the sample in our study was approximately 4.5 times larger. Moreover, comparing our infection rate to another retrospective study of 341 patients which was exploring the surgical outcome between surgical matricectomy and phenol matricectomy, there was $2.9 \%$ rate of infection among 139 patients who received phenol matricectomy (38). Their findings may not be directly comparable to our studies as they used patients as subjects, whereas procedures were the sample population in this study. Although they mentioned 520 procedures were performed on 341 patients, it was unclear as to the total number of procedures received in each group. Interestingly, it has also been reported that the safety profile of phenol decreases as application time increases (17). The mean complete healing time, mean duration of drainage and duration of tissue damage was found to be statistically significant shorter in 1-minute phenol application than 2 and 3-minute phenol applications $(p<0.001)(17)$. One should be mindful that the primary outcomes used in the study were tissue damage and drainage, which may be postoperative sequalae of phenolisation rather than infection (17). Therefore, the incidence of infection following different phenol application time remain uncertain.

Another valuable finding that has arisen was with regards to the predominant age group of postoperative infection. In this study, the most frequent incidents fell between the ages of 41-64. This finding is discordant with an existing retrospective study that found infection rate significantly increases in patients age $>60(p<0.01)(2)$. However, Terrill et al.'s results may be questionable as they appear to be older patients who underwent radical excision, an invasive procedure for management of onychocryptosis. Furthermore, Terrill et al.'s study found no statistically significant relationship between partial nail avulsion and postoperative infection rate $(p=0.78)$. The definition of office setting was unclear which may be due to how this study was carried out in Australia where the healthcare system is different (2). 
Therefore, it is questionable if the results of their study could be extrapolated to a wider population, let alone to a different countries population.

All 31 procedures within this study which presented with postoperative infection were prescribed with antibiotics, with $100 \%$ successful treatment rate. The incidence and effectiveness of preoperative antibiotics usage in this study were not assessed as the objective was not to explore the impact preoperative usage of antibiotics in procedure outcome. Although current evidence does not support the use of routine preoperative antibiotic prophylaxis in minor procedures for onychocryptosis, postoperative infection can lead to detrimental consequences (39). An unfortunate example would be postoperative surgical site infection resulting in gangrene and subsequent amputation, even in a healthy individual (40). However, current study suggests that the risk for this should be low. Among the cases which presented with postoperative infection in our study $(\mathrm{N}=31), 51.6 \%$ of them $(\mathrm{N}=16)$ had background medical issues. For example, endocrine and metabolic disorders, autoimmune disease, cardiovascular disease. The remainder were of good general health $(\mathrm{N}=15)$. However, there is no study to date that has found a statistically significant relationship between postoperative infection following nail surgery and presence of chronic diseases. Furthermore, there is a known increased risk of postoperative infection leading to toe amputation in patients who are active smokers (41). Although the study focuses on the incisional method in managing onychocryptosis, the findings should be applied cautiously to non-incisional techniques to prevent uncommon complications (41). Only one patient who found to have postoperative infection reported being an active smoker. However, active smokers are reported to have a 4.3 times increased risk of developing postoperative complications following foot surgery compared to non-smokers whereas patients with a smoking history were 1.9 times more likely to have complications following foot surgery (42). The clinical implication is that despite the factors affecting postoperative infection remains unclear, a holistic approach which considers these variables is essential when making clinical judgements and treatment recommendations in order to achieve optimal patient outcomes.

\section{Strengths and limitations of the study}

As this study adopted a convenience sample, it could mean that the selection bias is minimised because all procedures within the designated timeframe were included following a robust criterion (43). Conversely, it could be possible that the results may not be able to be extrapolated to other departments/Trusts. However, all routine minor procedure should only be performed in ASA 1 or 2 grading, and should be avoided in ASA 3 patients (3). It could be argued that the sampling method in this study is appropriate and the findings should be generalisable to community podiatry departments which routinely perform non-invasive minor procedure. Furthermore, this study aimed to obtain a snapshot of the effectiveness of this procedure in a community podiatry setting. Retrospective designed studies are categorised as non-experimental studies where they are weak in nature to draw causal relationships (44). However, the results of this study could be considered to set as a good benchmark for both internal and multicentre research development. 
This study included 272 participants, of which 72 participants had two procedures and 5 participants had three procedures performed in one sitting, resulting in a total of 354 procedures performed. It is acknowledged that power calculation was not performed prior to study commencement; however, it was felt that this calculation was not required as the sample size over the pre-determined time frame is established. Although the group numbers may be undersized, the findings appear to correlate with existing research. There have been recommendations that a sample size of 300 is the minimum amount to be considered adequate in quantitative research for factor analysis (45). We would thus argue that as a consequence, our sample size is sufficient to draw meaningful conclusions from.

Due to the nature of electronic patient records, the quality of the data being analysed is intrinsically linked to the individual clinician's comprehensiveness and accuracy documentation (46). Nevertheless, they will remain easy to obtain and with minimal risk of misinterpretation for any future comparisons (47). It is also possible that the risk of researcher bias is reduced as the entries are constructed without a dedicated research purpose in mind (46). Furthermore, the time period over which the study encompasses, especially on nail regrowth requires justification. Our retrospective period (the last procedure included was in December 2019 to allow 15 months to elapse) could be considered too short to provide enough data to show the true incidence of recurrence as literature has shown that the recurrence can happen up to 17 months postoperatively (36). It is acknowledged that the ideal retrospective time period could be longer than 15 months to observe the reoccurrence of symptoms; however, due to the change of the local service provider and the electronic software system, the time period available to us was unamendable. Whilst the time period of 15 months was allowed to observe the reoccurrence of symptoms, it can nevertheless be argued that clinically relevant recurrence would occur within this time period irrespectively (7).

Moreover, the most complete picture of postoperative infection may not have been captured as this was identified through patient records within the community setting only. For example, valid negative outcomes would not be captured by the techniques employed here if the patient consulted their general practitioner in event of a possible infection to obtain antibiotics and did not request a review appointment with podiatry or mention this in any follow-up appointments. Nevertheless, this would only cause an underestimation of the postoperative complications and our findings would thus at a minimum be the best-case scenario. Furthermore, It has been well discussed in the literature that postoperative inflammation following phenolisation is common as phenol is a caustic agent (28). It could even be considered that if general practitioners as opposed to podiatrists may be more inclined to misdiagnose the postoperative presentation and could have subsequently prescribed antibiotics for a postoperative sequalae rather than a true infection. Finally, whilst it is possible to review the patient records by the general practitioner, due to the time constraint and single authorship of this project, it was deemed not feasible.

Another limitation of this study is that the results are based on observations, followed by interpretation and conclusions drawn from them. It is arguable that this approach does not capture a full picture of the overall effectiveness of the minor procedure as it excludes patient's satisfaction following the procedure. The addition of incorporating investigation into patient's satisfaction may allow further exploration of the 
effectiveness of the treatment, which on certain aspects, observations alone could not have revealed (46). A mixed method study may be beneficial for further research as this is considered a more robust research based on the hierarchy evidence and possibly could provide further insight into the circumstances surrounding factors in decision making and clinical commissioning of patient care (44).

\section{Conclusion}

This study has demonstrated the relative effectiveness of minor surgery for onychocryptosis within a community podiatry department. Further research needs to be conducted before department specific conclusions about phenol application duration and recurrence rate can be accurately and reliably drawn. The findings should be used cautiously, and a holistic approach is paramount when making clinical judgements and treatment recommendations.

With the expansion of podiatry as one of the core teams within the primary care setting, this specialty is essential in offloading workload from general practitioners, who historically used to be the main source in providing this type of care. It is important to be able to demonstrate effective, safe and quality delivery of services to the clinical commissioning group of this specialty within the podiatry department. This can be achieved by providing quality metrics in both objective and subjective outcome measures. The current figures yielded by this study provide quantitative data that can be used to showcase its effectiveness in clinical practice to the commissioners responsible for organising care within the locality.

There appears to be gaps in high quality prospective research in this area of the specialty. Further investigation into this area of expertise is recommended to improve patient outcomes. Specifically, randomized controlled trials and prospective studies to investigate the treatment outcomes of surgical management of onychocryptosis in order to demonstrate causative links with respect to the important variables such as duration of application and chronic medical conditions. These studies would be of benefit to clinicians and their future decision making.

\section{Abbreviations}

ASA: American Society of Anaesthesiologists; HCPC: Health Care Professional Council; PASCOM-10: Podiatric and Surgical Clinical Outcome Measurement

\section{Declarations}

\section{Ethics approval and consent to participate}

Ethical approval was obtained from Course Ethics Panel of the M.Sc. Health Studies, Department of Nursing and Midwifery, School of Human and Health Sciences, University of Huddersfield. This is also registered as a clinical audit within the Quality Improvement Department (1190).

\section{Consent for publication}


Not Applicable.

\section{Competing interests}

The authors declare that they have no competing interests.

\section{Funding}

This study did not receive any specific grant from the funding agencies in the public, commercial, or notfor-profit sectors.

\section{Author's contributions}

All the authors read and approved the final manuscript. SXT: Study design, literature search, data collection, data analysis, draft manuscript. AB: Manuscript draft \& review. SHI: Manuscript review.

\section{Acknowledgements}

The main author would like to thank academic supervisor for guidance in writing this study.

\section{References}

1. Vural S, Bostanci S, Koçyigit P, Çaliskan D, Baskal N, Aydin N. Risk Factors and Frequency of Ingrown Nails in Adult Diabetic Patients. J Foot Ankle Surg [Internet]. 2018;57(2):289-95. Available from: https://doi.org/10.1053/j.jfas.2017.10.006

2. Terrill AJ, Green KJ, Salerno A, Butterworth PA. Risk factors for infection following ingrowing toenail surgery: a retrospective cohort study. J Foot Ankle Res. 2020;13(1):1-9.

3. College of Podiatry. Nail Surgery Guidelines. Vol. 2, College of Podiatry. 2019.

4. Pico AMP, Verjano E, Mayordomo R. Relation between nail consistency and incidence of ingrown toenails in young male runners. J Am Podiatr Med Assoc. 2017;107(2):137-43.

5. Imai A, Takayama K, Satoh T, Katoh T, Yokozeki H. Ingrown nails and pachyonychia of the great toes impair lower limb functions: Improvement of limb dysfunction by medical foot care. Int J Dermatol. 2011; $50(2): 215-20$.

6. Moustaide K. Controlled trial comparing the efficacy of $88 \%$ phenol versus $100 \%$ trichloracetic acid for chemical matricectomy in the management of ingrown toenail. Our Dermatology Online. 2019;10(2):116-20.

7. Eekhof J, Van Wijk B, Knuistingh Neven A, Van Der Wouden J. Interventions for ingrowing toenails. Cochrane Libr. 2012;(4).

8. Stewart CR, Algu L, Kamran R, Leveille CF, Abid K, Rae C, et al. Patient Satisfaction with Treatment for Onychocryptosis: A Systematic Review. Ski Appendage Disord. 2020;6(5):272-9. 
9. Krishan R, Latham A, Barlow-Kearsley E, Hodder S. Evaluating Clinical Outcomes of Nail Surgery: A Retrospective Audit of 80 Patients Using PASCOM-10. Pod Now. 2016;10-3.

10. Chang HC, Lin MH. Comparison of chemical matricectomy with trichloroacetic acid, phenol, or sodium hydroxide for ingrown toenails: A systematic review and network meta-analysis. Acta Derm Venereol. 2020;100(4):1-2.

11. Akkus A, Demirseren DD, Demirseren ME, Aktas A. The treatment of ingrown nail: Chemical matricectomy with NAOH versus wedge resection. Dermatol Ther. 2018;31(5):1-5.

12. Espensen EH, Nixon BP, Armstrong DG. Chemical matrixectomy for ingrown toenails: Is there an evidence basis to guide therapy? J Am Podiatr Med Assoc. 2002;92(5):287-95.

13. André MS, Caucanas M, André J, Richert B. Treatment of Ingrowing Toenails With Phenol $88 \%$ or Trichloroacetic Acid 100\%: A Comparative, Prospective, Randomized, Double-Blind Study. Dermatol Surg. 2018;44(5):645-50.

14. Talwar A, Puri N. A study on the surgical treatment of ingrowing toenail with nail excision with chemical matricectomy versus nail excision alone. J Pakistan Assoc Dermatologists. 2012;22(4):350-3.

15. Pérez-Rey J, Mediavilla-Saldana L, Martínez-Nova A. Exploring postoperative outcomes for ingrown toenails. naoh vs wedge resection techniques. Dermatologic Surg. 2014;40(3):281-7.

16. Montesi S, Lazzarino Al, Galeone G, Palmieri R, Montesi M. The Recurrence of Onychocryptosis when Treated with Phenolization: Does Phenol Application Time Play a Role? A Follow-Up Study on 622 Procedures. Dermatology. 2019;235(4):323-6.

17. Tatlican S, Yamangokturk B, Eren C, Eskioglu F, Adiyaman S. Comparison of phenol applications of different durations for the cauterization of the germinal matrix: An efficacy and safety study. Acta Orthop Traumatol Turc. 2009;43(4):298-302.

18. Modha MRK, Morriss-Roberts C, Smither M, Larholt J, Reilly I. Antibiotic prophylaxis in foot and ankle surgery: A systematic review of the literature. J Foot Ankle Res. 2018;11(1):1-14.

19. Grover C, Khurana A, Bhattacharya SN, Sharma A. Controlled trial comparing the efficacy of $88 \%$ phenol versus $10 \%$ sodium hydroxide for chemical matricectomy in the management of ingrown toenail. Indian J Dermatol Venereol Leprol. 2015;81(5):472-7.

20. Terzi E, Güvenç U, Türsen B, Tayfun E, Cihan Çoşansu N, Türsen Ü. Comparison of Phenol and Trichloroacetic Acid Chemical Matricectomies in the Treatment of Unguıs Inkarinatus. SM Dermatology J. 2018;4(1):1-4.

21. Gerrish K, Lathlean J. The research process in nursing. John Wiley \& Sons, Incorporated; 2015.628 p.

22. Ozawa T, Nose K, Harada T, Muraoka M, Ishii M. Partial matricectomy with a CO2 laser for ingrown toenail after nail matrix staining. Dermatologic Surg. 2005;31(3):302-5.

23. Cho SY, Kim YC, Choi JW. Epidemiology and bone-related comorbidities of ingrown nail: A nationwide population-based study. J Dermatol. 2018;45(12):1418-24. 
24. Tobin DJ. Introduction to skin aging. J Tissue Viability [Internet]. 2017;26(1):37-46. Available from: http://dx.doi.org/10.1016/j.jtv.2016.03.002

25. Orentreich N, Markofsky J, Vogelman JH. The effect of aging on the rate of linear nail growth. J Invest Dermatol. 1979;73(1):126-30.

26. PASCOM. 2019 PASCOM National Data Report. Podiatric surgery, nail surgery and injection therapies [Internet]. 2019. Available from: https://live.pascom-10.com/report/html/470077c7-ece1-09c1-da7d$08 d 7 c 5 a 380 c b$

27. PASCOM. 2018 PASCOM National Data Report. Podiatric surgery, nail surgery and injection therapies. 2018.

28. Muriel-Sánchez JM, Becerro-de-Bengoa-Vallejo R, Montaño-Jiménez P, Coheña-Jiménez M. The Treatment of Ingrown Nail: Chemical Matricectomy With Phenol Versus Aesthetic Reconstruction. A Single Blinded Randomized Clinical Trial. J Clin Med. 2020;9(3):845.

29. Haneke E. Controversies in the treatment of ingrown nails. Dermatol Res Pract. 2012;2012.

30. Boberg JS, Frederiksen MS, Harton FM. Scientific analysis of phenol nail surgery. J Am Podiatr Med Assoc. 2002;92(10):575-9.

31. Aveyard H, Sharp P. A Beginner's Guide to Evidence Based Practice in Health and Social Care. 3rd ed. McGraw-Hill Education. 2017. 258 p.

32. Becerro De Bengoa Vallejo R, Losa Iglesias ME, Viejo Tirado F, Serrano Pardo R. Cauterization of the germinal nail matrix using phenol applications of differing durations: A histologic study. J Am Acad Dermatol [Internet]. 2012;67(4):706-11. Available from: http://dx.doi.org/10.1016/j.jaad.2012.05.002

33. Brown AM, Kaplan LEOM, Brown ME. Phenol-induced histological skin changes: hazards, technique and uses. Br J Plast Surg. 1960;13:158-69.

34. Cordoba Diaz D, Losa Iglesias ME, Cordoba Diaz M, Becerro De Bengoa Vallejo R. Evidence of the efficacy of alcohol lavage in the phenolization treatment of ingrown toenails. $J$ Eur Acad Dermatology Venereol. 2011;25(7):794-8.

35. Vaccari S, Dika E, Balestri R, Rech G, Piraccini BM, Fanti PA. Partial excision of matrix and phenolic ablation for the treatment of ingrowing toenail: A 36-month follow-up of 197 treated patients. Dermatologic Surg. 2010;36(8):1288-93.

36. Bostanci S, Ekmekçi P, Gürgey E. Chemical matricectomy with phenol for the treatment of ingrowing toenail: A review of the literature and follow-up of 172 treated patients. Acta Derm Venereol. 2001;81(3):181-3.

37. Shi J, Lv Z, Nie M, Lu W, Liu C, Tian Y, et al. Human nail stem cells are retained but hypofunctional during aging. J Mol Histol [Internet]. 2018;49(3):303-16. Available from: http://dx.doi.org/10.1007/s10735-018-9769-0

38. Romero-Pérez D, Betlloch-Mas I, Encabo-Durán B. Onychocryptosis: a long-term retrospective and comparative follow-up study of surgical and phenol chemical matricectomy in 520 procedures. Int $\mathrm{J}$ Dermatol. 2017;56(2):221-4. 
39. Córdoba-Fernández A, Ruiz-Garrido G, Canca-Cabrera Á. Algorithm for the management of antibiotic prophylaxis in onychocryptosis surgery. Foot. 2010;20(4):140-5.

40. Noula AGM, Tochie JN, Tchuenkam LW, Abang DA, Essomba R. Surgical site infection leading to gangrene and amputation after ambulatory surgical care of an ingrown toenail: A case report. Patient Saf Surg. 2019;13(1):1-7.

41. Adkeniz H, Ozer K, Dikmen A, Kocer U. Common surgery, uncommon complication. Dermatol Pract Concept. 2015;5(4):5-7.

42. Bettin CC, Gower K, McCormick K, Wan JY, Ishikawa SN, Richardson DR, et al. Cigarette smoking increases complication rate in forefoot surgery. Foot Ankle Int. 2015;36(5):488-93.

43. Gerrish K, Lacey A, Cormack D. The research process in nursing. 6th ed. John Wiley \& Sons, Incorporated. 2010.

44. Polit DF, Beck CT. Nursing research: generating and assessing evidence for nursing practice. Eleventh e. Philadelphia Wolters Kluwer. Philadelphia Wolters Kluwer; 2021. 839 p.

45. Tabachnick BG, Fidell LS. Using Multivariate Statistics. Sixth edit. Pearson. 2014.

46. Moule P, Aveyard H, Goodman ML. Nursing research: an introduction. Vol. 3rd ed, London: SAGE. 2017.

47. Jolley J. Introducing research and evidence-based practice for nursing and healthcare professionals. Third ed. Routledge. 2020.

\section{Figures}

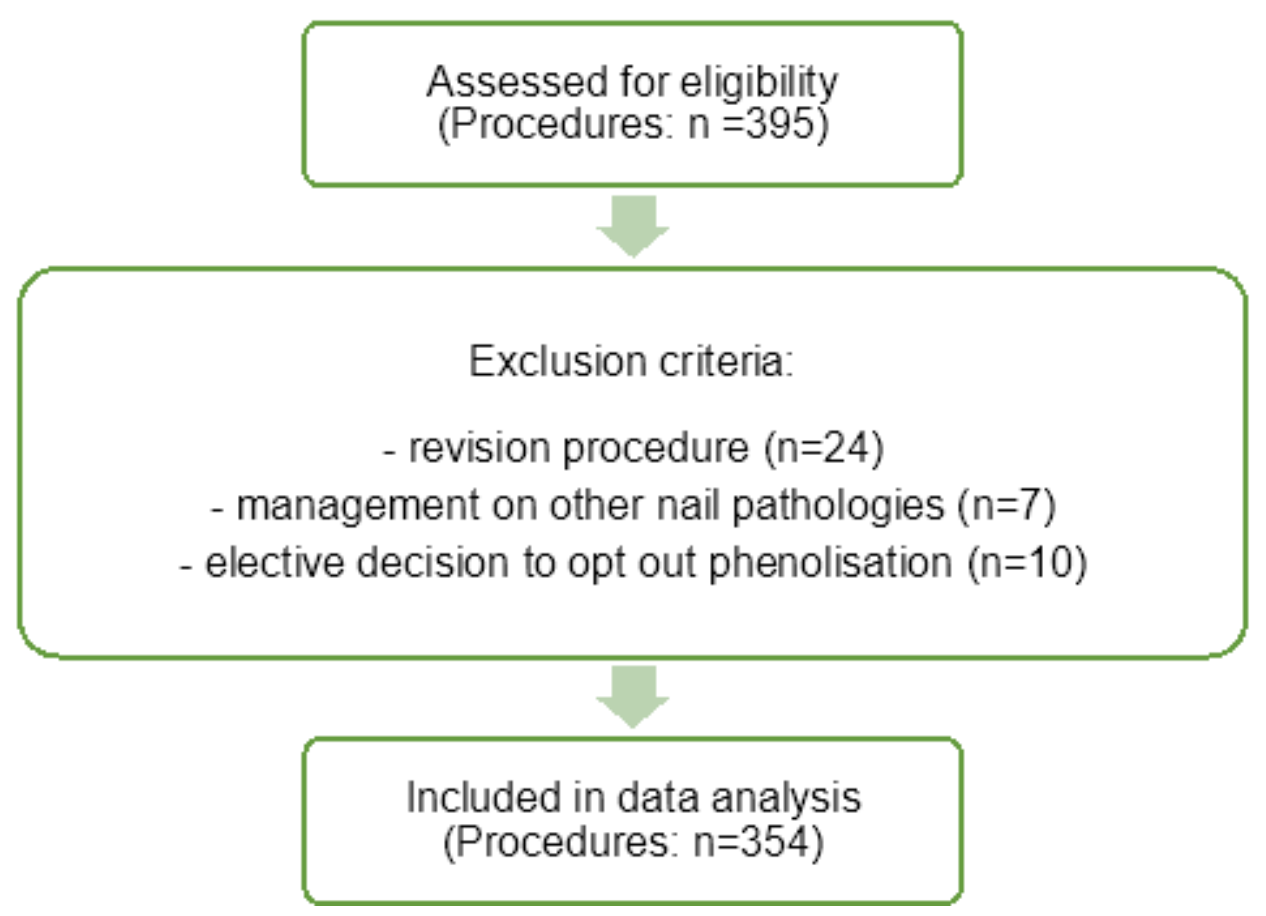

Figure 1 
Study flow chart

\section{Figure 2: Breakdown of relevant medical conditions ( $\mathrm{N}=107)$}

- Endocrine and metabolic disorder

- Cardiovascular disease

- Endocrine/metabolic disorder and cardiovascular disease

- Autoimmune disease

- Endocrine/metabolic disorder and autoimmune disease

- Pulmonary disease

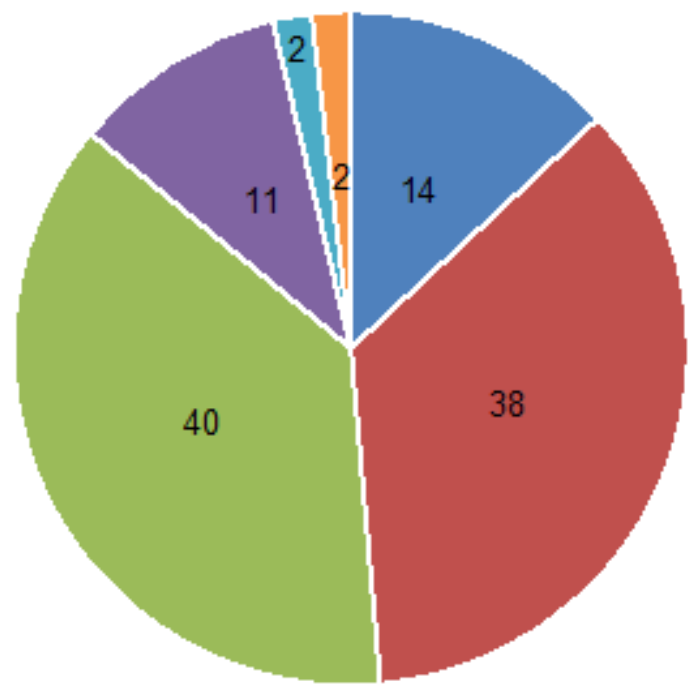

Figure 2

See image above for figure legend

Figure 3: Surgical site for partial nail avulsion $(\mathrm{N}=300)$

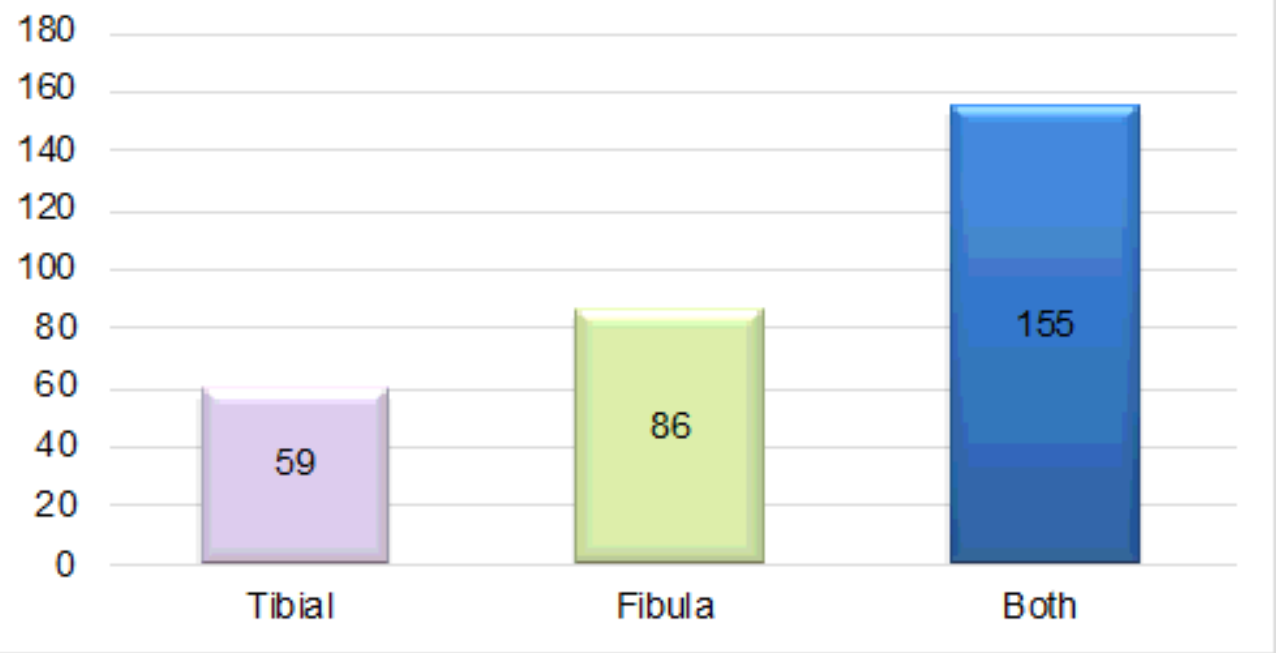

Figure 3

See image above for figure legend 
Figure 4: Time taken for identification of recurrence of the nail, months $(\mathrm{N}=20)$

\section{5}

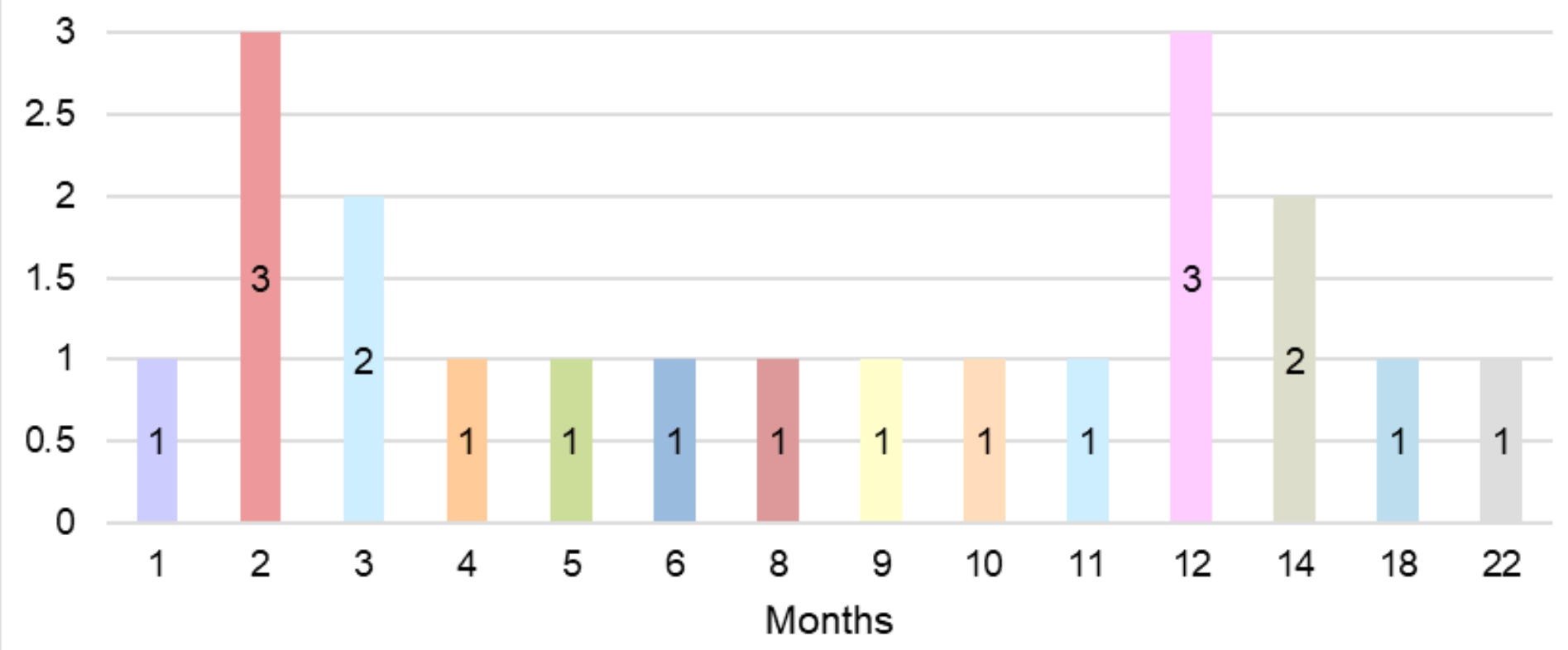

Figure 4

See image above for figure legend 


\section{Figure 5: Age distribution of patients with a recurrence $(\mathrm{N}=20)$}

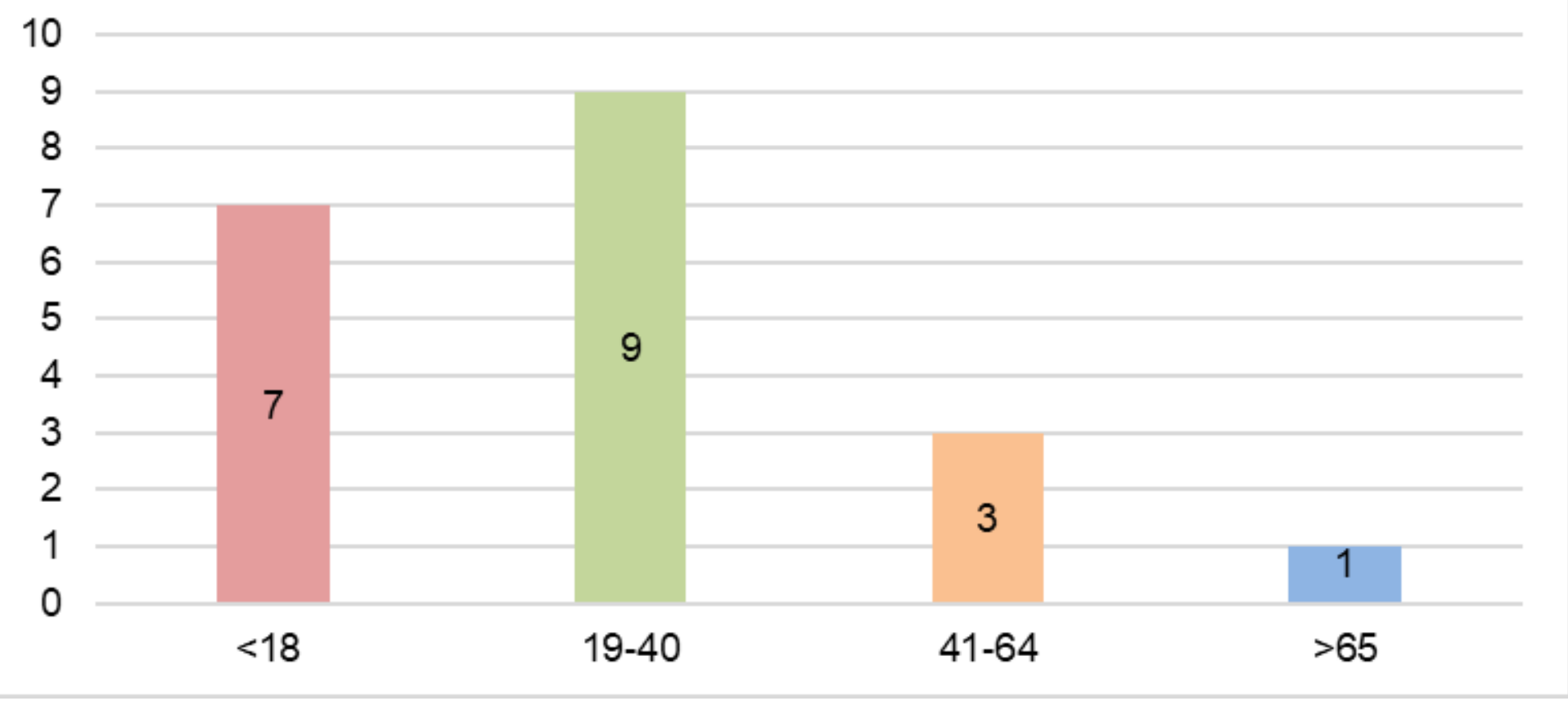

Figure 5

See image above for figure legend 


\section{Figure 6: Age distribution of procedures with postoperative infection $(\mathrm{N}=31)$}

18

16

14

12

10

8

16

6

4

2

0

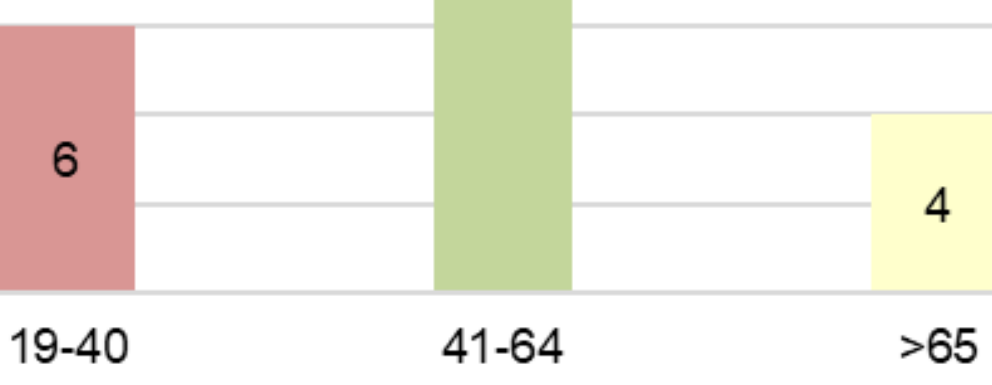

Figure 6

See image above for figure legend 


\section{Figure 7: Frequency of relevant medical conditions present in patients with postoperative infection $(\mathrm{N}=16)$}

\section{7}

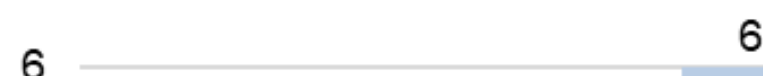

6

5

4

4

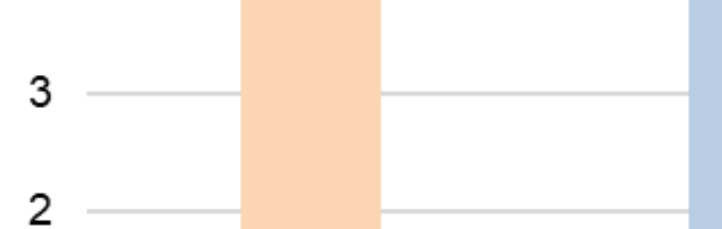

2

1

0

Endocrine/metabolic Endocrine/metabolic Autoimmune disease Cardiovascular disease disorder

6

3

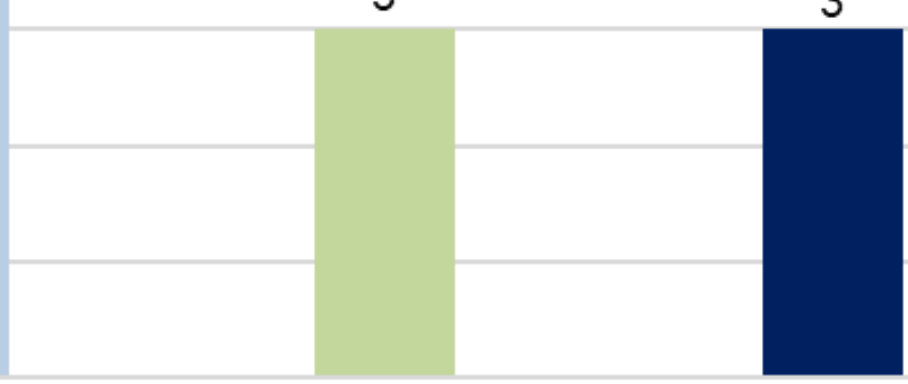
cardiovascular disease

Figure 7

See image above for figure legend

\section{Supplementary Files}

This is a list of supplementary files associated with this preprint. Click to download.

- Onlinefloatimage7.png 